\title{
A Two-Dimensional Central Non-Oscillatory Scheme for Inviscid Compressible Flows
}

\author{
Gregorio Gerardo Spinelli ${ }^{1}$, Bayram Celik ${ }^{1}$ \\ ${ }^{1}$ Istanbul Technical University \\ Department of Astronautical Engineering \\ 34469 Istanbul, Turkey \\ spinelli15@itu.edu.tr; celikbay@itu.edu.tr
}

\begin{abstract}
For this study, we developed a two-dimensional high order finite volume method code to solve inviscid compressible flows. In particular, the central essentially non-oscillatory (CENO) scheme improves spatial reconstruction, and the Runge-Kutta $4^{\text {th }}$ order scheme enhances temporal accuracy. The CENO scheme provides a smoothness indicator that dictates whether a cell is under-resolved. One can evaluate this indicator either with the first or second layer of each cell's neighbours, where it is applicable. For under-resolved cells, the reconstruction switches to limited second order instead of high order. We solved the shock tube in terms of accuracy and computational time for different spatial high order reconstructions. Results showed that for problems which present discontinuities, the usage of high order schemes to enhance spatial reconstruction does not improve accuracy as expected. Furthermore, the presence of discontinuities effects also the order of convergence of the solver. Specifically the order of convergence for all the studied spatial reconstructions is always as first order.
\end{abstract}

Keywords: finite volume method, central essential non-oscillatory scheme, inviscid flows, compressible flows

\begin{tabular}{|l|l|l|l|}
\hline \multicolumn{2}{|c|}{ Nomenclature } & Symbol & Description \\
\hline Symbol & Description & \\
$A$ & & Greek & \\
$\#$ Cells & Matrix of geometrical coefficients & $\alpha$ & Smoothness parameter \\
$D$ & Number of cells of the central stencil & $\gamma$ & Ratio of specific heats \\
$\# D$ & Unknown reconstruction coefficients & $\rho$ & Density \\
$E$ & Number of dimensions & $\Omega$ & Volume \\
$g$ & Total energy & Vectors & \\
$\bar{g}$ & Generic variable & $\overrightarrow{F_{c}}$ & Convective fluxes \\
$H$ & Cell average quantity of g & $\vec{W}$ & Conservative variables \\
$k$ & Total Enthalpy & & \\
$M$ & Order of accuracy & Subscript & \\
$N$ & Number of neighbors & Minimum value \\
$N_{f}$ & Number of unknown D & \\
$p$ & Number of faces of an element & Superscript & \\
$p_{i}$ & Pressure & General index \\
$S$ & CENO general index & $n$ & Quantity value at time $n$ \\
$S c$ & Smoothness indicator & Math Signs & \\
$t$ & Threshold for S & Increment \\
$u$ & Time & \\
$v$ & Horizontal component of velocity vector & $\Delta$ & \\
$V$ & Vertical component of velocity vector & & \\
\hline
\end{tabular}




\section{Introduction}

Modern engineering applications trust Computational Fluid Dynamics, CFD to obtain realistic solution of high complex problems. The advent of new technologies enhanced computer power that in turn boosted CFD application for such problems. Lewis Fry Richardson was one of the first pioneer who applied CFD to weather forecast, in 1922 [1]. Although he dramatically failed, he applied the same concepts we are adopting for modern CFD, such as approximation through finite differences and dividing the domain in cells. A few decades later, in 1960s, Harlow performed several two-dimensional CFD analyses taking into consideration the Euler equations for unsteady compressible flows [2]. Modern CFD applications consists of three-dimensional domains where the full Navier-Stokes equations model the fluid flow, such as blade cooling [3], lift-drag predictions [4], shock wave interactions [5], etc.

To resolve a complex flow field accurately, one is obligated to mesh the domain densely. High order method in space and time have demonstrated the capabilities of resolving such flows with desired accuracy, by using coarser meshes [6]. In the literature, there are several schemes available, such as essentially non-oscillatory (ENO) schemes [7], Weighted ENO (WENO) schemes [7], Central ENO (CENO) schemes [8], discontinuous Galerkin (DG) schemes [9], and finite-difference (FD) schemes [9]. The main challenge of a high order scheme is the preservation of monotonicity during reconstruction stage. Within the ENO family, ENO, WENO, and CENO use a different technique to preserve monotonicity. In particular, ENO scheme is excluding from stencils those cells that contain a discontinuity, by reconstructing the solution on several stencils and choosing the one that provides the smoothest reconstruction [7]. In order to choose the most appropriate stencil for reconstruction, the WENO scheme applies a weighting factor to each stencil, which is depending upon the solution [7]. At last, the CENO scheme is using a central stencil to reconstruct the solution, but the order of accuracy is reduced to limited second order where the cells present a discontinuity of any kind [9]. To understand whether a cell is under resolved, we use a smoothness indicator [9]. Due to the high possibility of ENO and WENO schemes at selecting the proper stencil, their usage requires an extreme computational cost. In order to resolve this issue, the CENO scheme uses a fix central stencil. However, the lower computational load comes along with loss of uniform high accuracy within the whole domain. Researchers, such as Ivan et al. [9], proved that this local loss of accuracy is not nullifying the strength of high order accuracy in the whole domain; rather the convergence rate is still at the high order.

The objective of this paper is to use CENO schemes to accurately solve the shock tube problem. We are going to analyse the effect that the smoothness indicator has on the solution, in terms of accuracy, and the enhancement of high order reconstruction for unsteady flows, by using Runge-Kutta $4^{\text {th }}$ order scheme, a high order time-stepping scheme [10].

\section{Numerical Method}

This section introduces the numerical scheme we use for this study. The finite volume method is applied to discretise the Euler equations without external and body forces, as follows [11].

$$
\begin{gathered}
\vec{W}^{n+1}=\vec{W}^{n}-\frac{\Delta t}{\Omega} \sum_{i=1}^{N_{F}}\left(\overrightarrow{F_{c}}\right)_{i} \Delta S_{i} \\
\vec{W}=\left[\begin{array}{c}
\rho \\
\rho u \\
\rho v \\
\rho E
\end{array}\right] \quad \overrightarrow{F_{c}}=\left[\begin{array}{c}
\rho V \\
\rho u V+n_{x} p \\
\rho v V+n_{y} p \\
\rho H V
\end{array}\right] \\
V=\vec{V} \cdot \vec{n}=u n_{x}+v n_{y} \\
H=E+p / \rho \\
p=(\gamma-1) \rho\left(E-\frac{V^{2}}{2}\right)
\end{gathered}
$$


The Advection Upstream Splitting Method +up (AUSM+up) [12] is the method that we used to evaluate convective fluxes in Eq. 2. In order to evaluate the summation in Eq. 1, the CENO scheme reconstructs the fluxes at each Gauss quadrature points of the cells' interfaces [9]. To reduce error propagation between each time step, we use a Runge-Kutta $4^{\text {th }}$ Kutta $4^{\text {th }}$ order scheme [10].

\subsection{CENO Scheme}

To be able to reconstruct fluxes at each Gauss quadrature point with CENO scheme, we follow these three steps. Firstly, for each cell we perform a k-exact order reconstruction. Then, we evaluate the smoothness indicator for each cell. Finally, for the under resolved cells we use a limited second order reconstruction. The following formula performs the k-exact order reconstruction.

$$
g_{i}^{k}(x, y)=\sum_{p_{1}=0}^{k} \sum_{p_{2}=0}^{k}\left(x-x_{i}\right)^{p_{1}}\left(y-y_{i}\right)^{p_{2}} D_{p_{1} p_{2}}, \quad p_{1}+p_{2} \leq k
$$

In Eq. $6 g_{i}^{k}$ represents a generic variable to be reconstructed at a point $(x, y)$, cell centre has the following coordinates $\left(x_{i}, y_{i}\right)$, and $D_{p_{1} p_{2}}$ represents the unknown coefficients. The number $\mathrm{N}$ of unknown coefficients depends on the dimensions \#D of the problem and the order of reconstruction $k$ required, as follows.

$$
N=\frac{1}{\# D !} \prod_{n=1}^{\# D}(k+n)
$$

The least square method evaluates the unknown coefficients for the given fix central stencil. The number of unknown $\mathrm{N}$ dictates the minimum number of cells inside the stencil. As can be seen from Fig. 1, for a 2D structured mesh, the single cell has 8 neighbours in the first layer (LV1) that are depicted in green, and 16 neighbours of second level (LV2) that are represented in red.

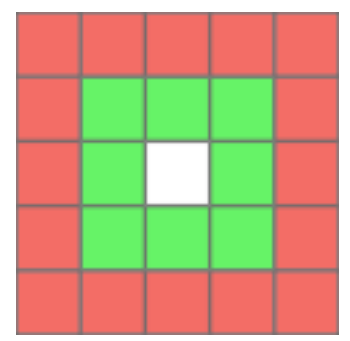

Fig. 1: Neighbours for a quad cell in 2D.

In order to let the least square problem have a proper amount of degree of freedom, we chose either 9 cells (LV1) or 25 (LV2) cells for the central stencil, based on the $\mathrm{N}$ value (see Tab.1).

Table 1: Number of cells that composes the central stencil.

\begin{tabular}{|c|c|c|c|}
\hline$k$ & $N$ & \#Cells_min $=N \cdot 1.5$ & \#Cells \\
\hline 1 & 3 & 4.5 & 9 \\
\hline 2 & 6 & 9 & 9 \\
\hline 3 & 10 & 15 & 25 \\
\hline 4 & 15 & 22.5 & 25 \\
\hline
\end{tabular}


To preserve the cell average quantity, $\overline{g_{l}}$ the CENO scheme enforces the least square reconstruction with the constraint.

$$
D_{00}=\bar{g}_{\imath}-\sum_{p_{1}=0}^{k} \sum_{p_{2}=0}^{k} D_{p_{1} p_{2}}\left(\overline{x^{p_{1}} y^{p_{2}}}\right)_{i}, \quad p_{1}+p_{2} \leq k
$$

The geometrical coefficient $\overline{x^{p_{1}} y^{p_{2}}}$ is evaluated as below.

$$
\left(\overline{x^{p_{1}} y^{p_{2}}}\right)_{i}=\frac{1}{\Omega_{i}} \iint_{\Omega_{i}}\left(x-x_{i}\right)^{p_{1}}\left(y-y_{i}\right)^{p_{2}} d A
$$

With the help of Equations 6, 8, and 9, the least square system takes the following form.

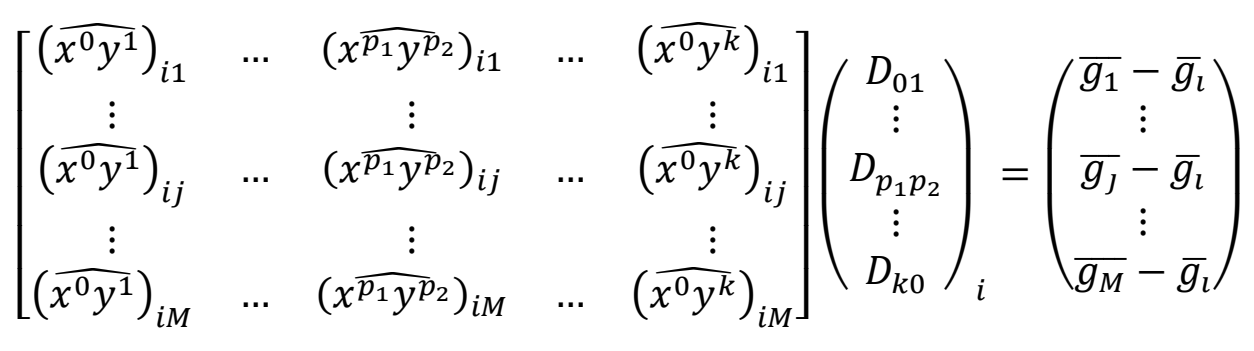

In Eq. 10, $M$ represents the number of neighbours of the cell $i, N$ represents the total amount of unknown coefficients minus one, and $D_{00}$ is set as a constraint. The elements of matrix $A$ are calculated as stated in Reference [13].

A smoothness indicator helps us decide whether a cell is under resolved. For CENO scheme, the following smoothness indicator is available in the literature $[9,13]$.

$$
S=\frac{\alpha}{\min \left(1-\alpha, 10^{-8}\right)} \frac{\# \text { Cells }-\mathrm{N}}{N-1}
$$

In the previous equation, $\alpha$ is determined as stated in [13]. One can evaluate the smoothness indicator with either just the first (green cells in Fig.1) or second layer (green and red cells in Fig.1) of cell's neighbours. Afterwards, the smoothness parameter is compared to a cut-off value $S c$, which is determined experimentally [13]. The range of variation of $S c$ is between 2000 and 5000. Thus, any $S$ value smaller than $S c$ determines the cell as under resolved. Finally, for under resolved cells a limited second order accurate reconstruction is performed, while a k-exact order reconstruction is adopted for the other cells. For this solver we use the Venkatakrishnan limiter [14] when reconstructing under resolved cells.

The CENO reconstruction is enforced at the boundaries. We apply simple boundary condition, such as inlet or outlet conditions, via ghost cells. While we apply complex boundary conditions, such as symmetric walls, by including the constraints at each gauss quadrature point into the least square matrix $A$. In particular, we apply a weight of $10^{3}$ to the constraints, and Eq. 8 is also included into matrix $A$ as weighted constraint [15].

We use an external C++ library, Eigen3.3.5 [16], to evaluate the least square solutions via the subroutine Householder QR factorization (A.colPivHouseholderQr().solve(b)).

\section{Results}

The problem analysed here is the shock tube problem. Initially, the domain is separated in two equal regions by a membrane as in Fig. 2. The region at the left of the membrane has an initial condition of a density equal to $1 \mathrm{Kg} / \mathrm{m}^{3}$ and 
a pressure equal to $1 \mathrm{~Pa}$. The initial condition for the other region is of a density equal to $0.125 \mathrm{Kg} / \mathrm{m}^{3}$ and a pressure equal to $0.100 \mathrm{~Pa}$.

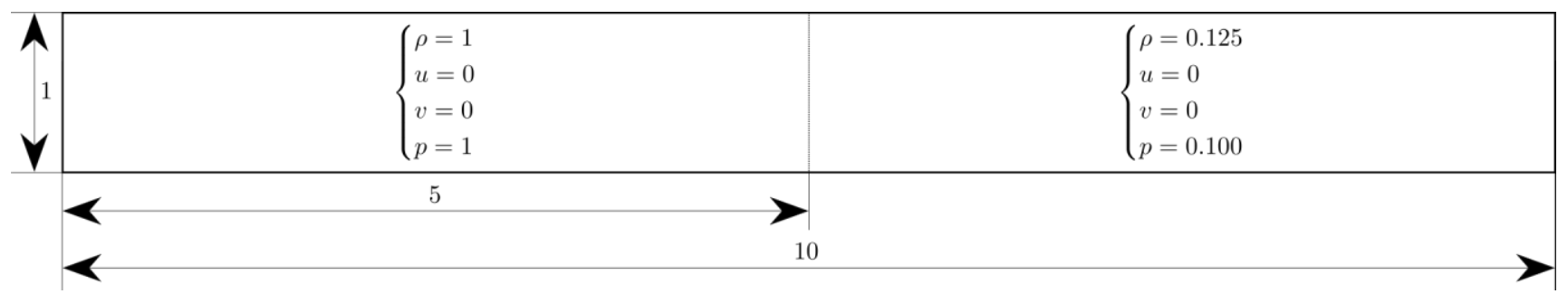

Fig. 2: Domain and initial conditions for the shock tube problem.

Fig. 3 shows our results for a fifth order spatial accuracy in terms of density distribution along the tube for different mesh sizes, from 500 cells though 10000 cells. The results are matching with the analytical solution [17]. As expected, when the mesh size increases the FVM results show better agreement with the analytic solution.

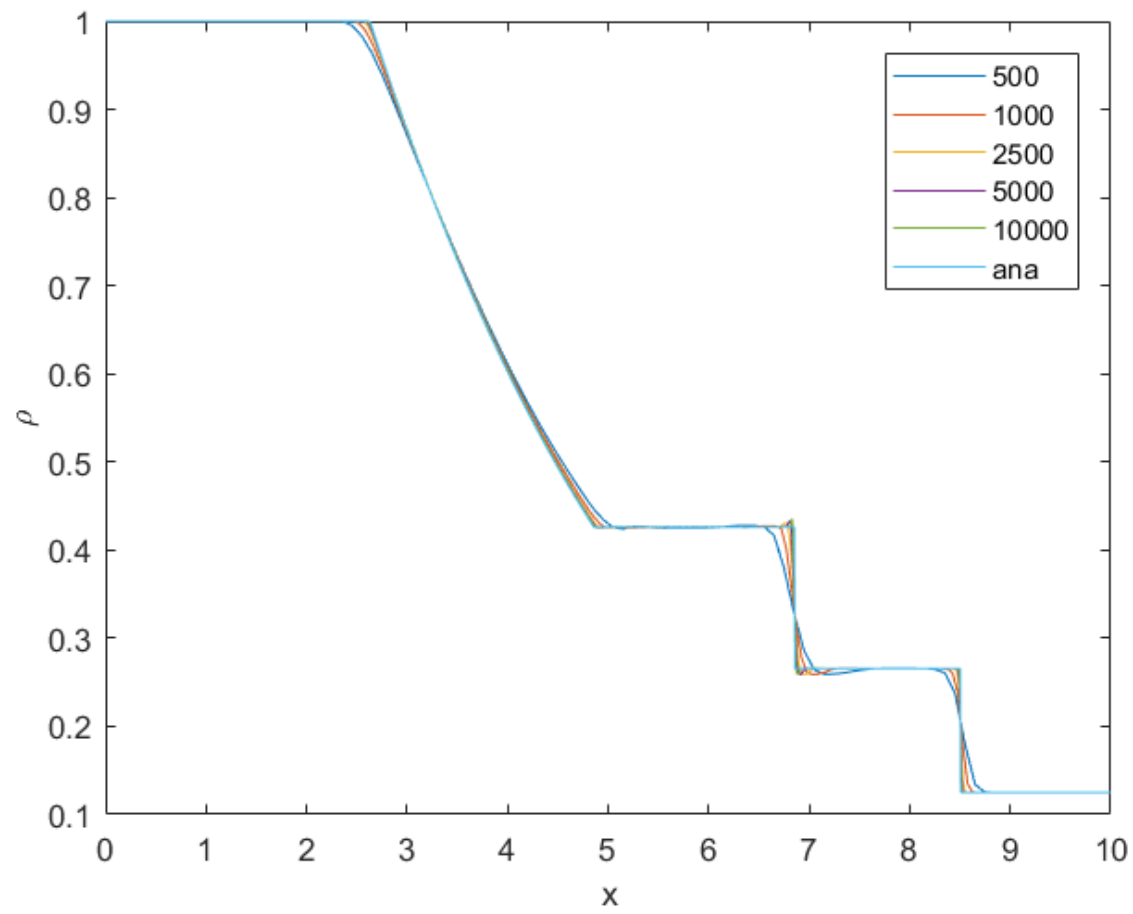

Fig. 3: Fourth order FVM results and the analytic solution in terms of density distribution along the tube.

Fig. 4 shows the L2 Norm of error in density with respect to the number of cells and the execution time. We evaluated the L2 Norm of error for different orders of spatial reconstruction (first, second, third, fourth and fifth). The lines labelled as LV1 indicate that the results are obtained with a smoothness indicator that is calculated with the fist layer of cell's neighbours. As can be seen from Fig.4, by switching from first order to second order of spatial reconstruction, there is a significant increase in the solution accuracy. However, the third, fourth and fifth order for the CENO scheme generate only a slightly increase of the accuracy. This discrepancy is due to the presence of discontinuities and strong shocks in the domain. For this problem, the presence of discontinuities also causes the order of convergence to be of first order for all k-exact order reconstruction, as shown in Fig. 4. The results obtained by evaluating the smoothness indicator only with first layer of cell's 
neighbours are less accurate than the corresponding results obtained by evaluating the smoothness indicator with the full central stencil.
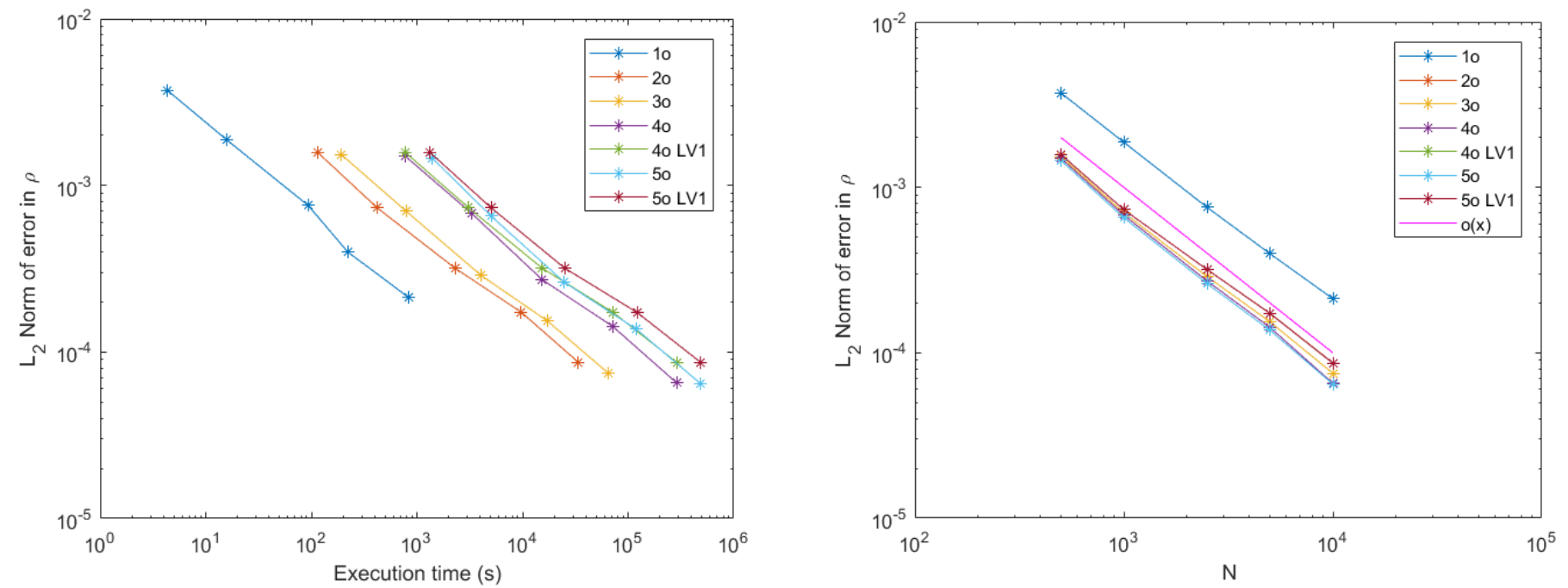

Fig. 4: Left and right figure show the L2 Norm of error in density with respect to execution time and mesh size, respectively for different order of spatial reconstructions.

\section{Conclusion}

For this study, we developed a two-dimensional high order FVM for inviscid compressible flows. The AUSM+up scheme evaluates the inviscid fluxes. The CENO scheme enhances the spatial accuracy, and the Runge-Kutta $4^{\text {th }}$ scheme enforces the temporal accuracy. For the CENO high order scheme, we compute the smoothness indicator for each cell either with the first layer of neighbours or with the second layer of neighbours.

The problem we solved is the shock tube. We obtained results for different orders of spatial reconstruction, and for the two different methods for the calculation of the smoothness indicator. Due to the presence of discontinuities in the domain, the order of convergence is of first order for all evaluated k-exact order reconstructions. The L2 Norm of error in density is showing that accuracy of second, third, fourth and fifth order schemes is slightly improving. However, switching from first order to second order highly improves the solution accuracy. The main difference between the two methods for computing the smoothness indicator is observed in the L2 Norm of error in density of the solution. Particularly by evaluating the smoothness indicator with the first layer of neighbours for fourth and fifth order of spatial accuracy, the L2 Norm of accuracy in density decreases to the one obtained with a second order spatial accuracy and the computational time decreases by nearly $0.82 \%$. The results suggest that to obtain solutions to problems that present discontinuities, a second order CENO scheme is a good candidate both in terms of accuracy and in terms of computational time.

In order to overcome those difficulties dictated by discontinuities, a possible solution is to couple CENO scheme with adaptive mesh refinement. Furthermore, the code can be extended to solve viscous compressible flows.

\section{Acknowledgements}

Gregorio Gerardo Spinelli would like to thank TUBITAK for Graduate Scholarship Program for International Students (Code 2215). Computing resources used in this work were provided by the National Center for High Performance Computing of Turkey (UHeM) under grant number 4006022019.

\section{References}

[1] O. F. Richardson, and S. Chapman, Weather prediction by numerical process, Dover Publications, 1965. 
[2] R. A. Gentry, R. E. Martin, and J. B. Daly, "An Eulerian differencing method for unsteady compressible flow problems", Journal of Computational Physics, vol. 1, no. 1, pp. 87-118, 1966.

[3] X. Fan, L. Li, J. Zou, J. Wang, and F. Wu, "Local heat transfer of vortex cooling with multiple tangential nozzles in a gas turbine blade leading edge cooling passage," International Journal of Heat and Mass Transfer, vol. 126-B, pp. 377$389,2018$.

[4] T. Lee, S. M. He, "The trailing vortices generated by a reverse delta wing with different wing configurations," Aerospace Science and Technology, vol.82-83, pp. 378-393, 2018.

[5] D. Knight, and M. Mortazavi, "Hypersonic shock wave transitional boundary layer interactions - A review," Acta Astronautica, vol. 151, pp. 296-317, 2018.

[6] S. Pirozzoli, "On the spectral properties of shock-capturing schemes," Journal of Computational Physics, vol. 219 , no. 2, pp. 489-497, 2006.

[7] Y. T. Zhang, and C. W. Shu, "Handbook of Numerical Analysis," Elsevier, 2016, pp. Pages 103-122.

[8] L. Ivan, and P. T. G. Clinton, "High-order solution-adaptive central essentially non-oscillatory (CENO) method for viscous flows," Journal of Computational Physics, vol. 257-A, pp. 830-862, 2014.

[9] M. Alhawwary, and Z.J. Wang, "Fourier analysis and evaluation of DG, FD and compact difference methods for conservation laws", Journal of Computational Physics, vol. 373, pp 835-862, 2018.

[10] L. Liu, X. Li, F. Q. Hu, "Nonuniform-time-step explicit Runge-Kutta scheme for high-order finite difference method," Computers \& Fluids, vol. 105, pp. 166-178, 2014.

[11] J. Blazek, Computational Fluid Dynamics, Butterworth-Heinemann, 2015.

[12] M. S. Liou, "A sequel to AUSM, Part II: AUSM+-up for all speeds," Journal of Computational Physics, vol. 214, no. 1, pp. 137-170, 2006.

[13] L. Ivan, "Development of High-Order CENO Finite-Volume Schemes with Block-Based Adaptive Mesh Refinement", Ph.D. dissertation, Department of Aerospace Science and Engineering, University of Toronto, 2010.

[14] V. Venkatakrishnan, "On the Accuracy of Limiters and Convergence to Steady State Solutions," in Proceedings of the $31^{\text {st }}$ Aerospace Sciences Meeting, 1993.

[15] C. L. Lawson, and R. J. Hanson, Solving least squares problems, Prentice-Hall, 1974.

[16] Eigen3.3.5 (2018, Dec 4) [Online]. Available: http://eigen.tuxfamily.org/index.php?title=Main_Page

[17] J. D. Anderson, Modern Compressible Flow, McGraw-Hill Education, 2002. 